\title{
Rare case of an aneurysmal bone cyst of the skull
}

\author{
Kaveh Akbari $^{1}$, Christine M. Fellner ${ }^{1}$ and Franz A. Fellner ${ }^{1,2 *}$ \\ ${ }^{1}$ Central Radiology Institute, Kepler University Hospital, Medical Faculty of the Johannes Kepler University, Linz, Austria \\ ${ }^{2}$ Medical Faculty of the Friedrich-Alexander-University of Erlangen-Nürnberg, Germany
}

We report a patient who was referred for assessment of obstructed nasal breathing being suspicious for sinusitis. Clinical examination showed deviation of the nasal septum to left, a nasal polyp on the right, and purulent secretions. Assessed laboratory parameters were normal (Figure 1).

Cranial computed tomography revealed a large (diameter $6 \mathrm{~cm}$ ) space-occupying mass involving the right maxillary sinus, sphenoid sinus, ethmoidal cells, and the nasal cavity. Adjacent bone structures were eroded. The osseous walls of the ethmoidal cells were abolished with thinning out the medial wall of the right orbit (Figure 2).

Further pre-surgical imaging with magnetic resonance visualized the expansive, polycyclic formation with honeycomb like appearance presenting with multiple fluid-fluid levels with different signal intensities. Parts of the cysts were bulging towards the temporal pole with reactive thickening of the dura. MR appearance was typical for an aneurysmal bone cyst. Diagnosis was confirmed by means of biopsy with consecutive histopathological work-up.

During this MR examination an contrast-enhanced MR angiography was acquired to evaluate possible involvement of the brain supplying arteries (Figure 3). The MRA demonstrated tight contact of the right internal carotid artery to the bone cyst with slight displacement of the artery by the mass effect of the aneurysmal bone cyst, but no stenosis.

Aneurysmal bone cysts (ABC) were first described by Jaffe und Lichtenstein in the year of 1942 [1]. They are benign expansive osteolytic bone tumors. The large blood-filled spaces are separated by connective

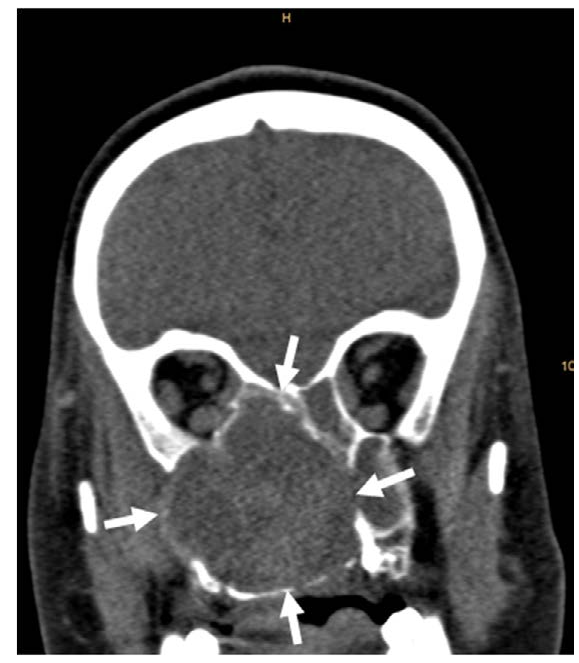

Figure 1. Computed tomography reveals a bone eroding mass (arrows) involving the nasal cavity and several paranasal sinuses. septa containing fibroblasts, osteoclastic giant cells and reactive woven bone. Cysts and septa are not outlined by endothelium. The most common age of onset is the second decade of life. There is no gender preference. Typical locations are the shaft of long bones, metaphysis of

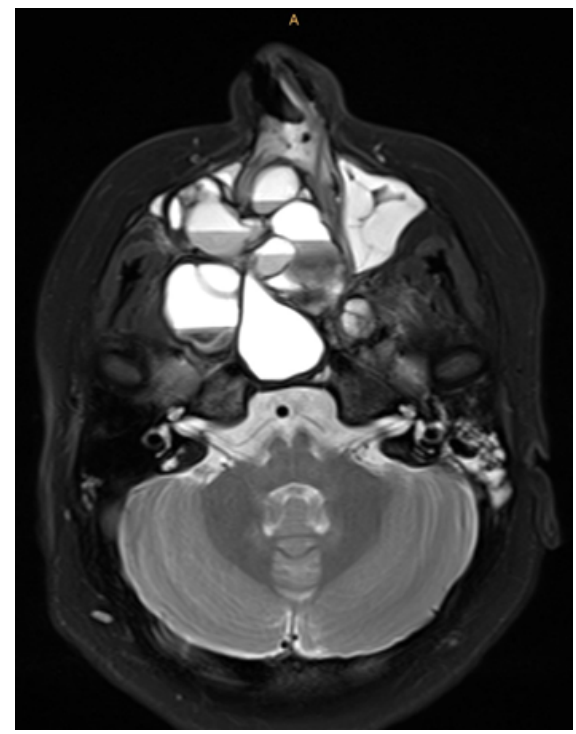

Figure 2. 1.5T MR examination: T2 fat suppressed image (transverse orientation) shows a typical honeycomb like appearance with multiple fluid-fluid levels.
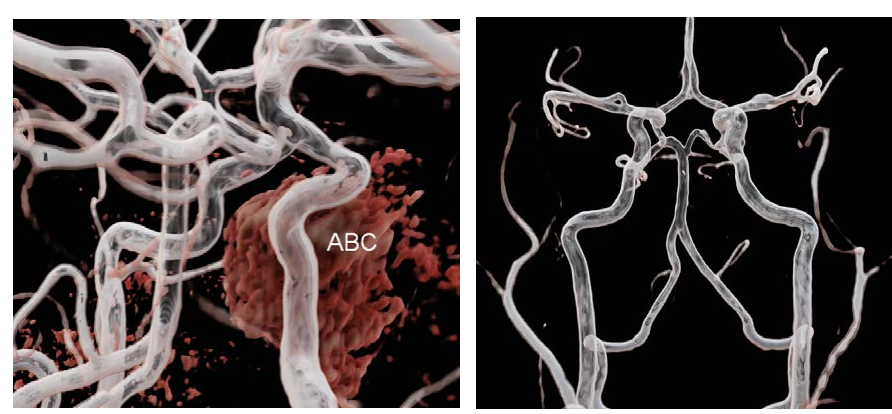

Figure 3. Contrast-enhanced MR angiography, 3D post processed with cinematic rendering. Tight contact of the right internal carotid artery (ICA) to the aneurysmal bone cyst with slight displacement of the ICA.

Correspondence to: Franz A. Fellner, Central Radiology Institute, Kepler University Hospital, Medical Faculty of the Johannes Kepler University, Linz, Austria, Medical Faculty of the Friedrich-Alexander-University of ErlangenNürnberg, Germany, E-mail: franz.fellner@kepleruniklinikum.at

Received: February 13, 2017; Accepted: March 06, 2017; Published: March 09, 2017 
posterior vertebrae and flat bones. Skull bones, however, are affected very rarely with an incidence of 3-6\% [2]. Honeycombing with fluidfluid levels on MR imaging is the typical appearance. In the case of a vast mass it is useful to visualize the vessels by means of MRA or CTA to evaluate the vascular situation (displacement, stenosis, topography of mass and adjacent vessels). In this case we used cinematic rendering [3-6], additionally to the standard MRA assessment procedures, to create also a clear and precise three-dimensional display of the massvessel topography.

Possible differential diagnoses of ABC include solitary bone cyst, giant cell carcinoma, osteoblastoma, hemangioma, chondroblastoma, chondromyxoid fibroma and teleangiectatic osteosarcoma.

\section{References}

1. Jaffe HL, Lichtenstein L (1942) Solitary unicameral bone cyst with emphasis on the roentgen picture, the pathologic appearance and the pathogenesis. Archives of Surgery 44: 1004-1025.

2. Yadav JS, Gupta AK, Kappar N (2011) Aneurysmal bone cyst of the paranasal sinuses a rare entity. Clinical Rhinology 4: 93-95.

3. Fellner FA (2016) Introducing cinematic rendering: a novel technique for postprocessing medical imaging data. J Biomedical Science and Engineering 9: 170-175.

4. Fellner FA (2016) "Cinematic rendering" of an Egyptian fish mummy with a fractured spinal column. Glob Imaging Insights 2: 1-2.

5. Fellner FA, Behawy M (2017) Cinematic and volume rendering of a scaphoid fracture. Glob Imaging Insights 2: 1-2.

Copyright: (C2017 Akbari K. This is an open-access article distributed under the terms of the Creative Commons Attribution License, which permits unrestricted use, distribution, and reproduction in any medium, provided the original author and source are credited. 